\title{
Troublesome aspects of the Renyi-MaxEnt treatment
}

\author{
A. Plastino, ${ }^{1,2,3}$ M. C. Rocca, ${ }^{1,2,4}$ and F. Pennini ${ }^{5,6}$ \\ ${ }^{1}$ Departamento de Física, Universidad Nacional de La Plata, Calle 49 s/n, 1900 La Plata, Buenos Aires, Argentina \\ ${ }^{2}$ Consejo Nacional de Investigaciones Científicas y Tecnológicas (IFLP-CCT-CONICET)-C. C. 727, 1900 La Plata, Argentina \\ ${ }^{3} S T h A R-E P F L$, Lausanne, Switzerland \\ ${ }^{4}$ Departamento de Matemática, Universidad Nacional de La Plata, Calle 50 s/n, 1900 La Plata, Buenos Aires, Argentina \\ ${ }^{5}$ Universidad Católica del Norte, Av. Angamos 0610, Antofagasta, Chile \\ ${ }^{6}$ Facultad de Ciencias Exactas y Naturales, Universidad Nacional de La Pampa, CONICET Peru 151, 6300 Santa Rosa, La Pampa, Argentina
}

(Received 14 February 2016; revised manuscript received 30 May 2016; published 27 July 2016)

\begin{abstract}
We study in great detail the possible existence of a Renyi-associated thermodynamics, with negative results. In particular, we uncover a hidden relation in Renyi's variational problem (MaxEnt). This relation connects the two associated Lagrange multipliers (canonical ensemble) with the mean energy $\langle U\rangle$ and the Renyi parameter $\alpha$. As a consequence of such relation, we obtain anomalous Renyi-MaxEnt thermodynamic results.
\end{abstract}

DOI: 10.1103/PhysRevE.94.012145

\section{INTRODUCTION}

The Renyi information measure $S_{R}$ is a generalization of those of both Hartley and Shannon, quantifying a system's diversity, uncertainty, or randomness. $S_{R}$ is an important quantity for several areas of scientific endeavor. One can mention, for instance, ecology, quantum information, the Heisenberg $X Y$ spin chain model, theoretical computer science, conformal field theory, quantum quenching, diffusion processes, etc. As a small sample, see for example, [1-10].

Information theory (IT) yields an extremely powerful inference approach, usually abbreviated as MaxEnt [11]. MaxEnt is able to describe quite general properties of arbitrary systems, in several areas of science, on the basis of scarce information. MaxEnt purports to provide one of the least-biased descriptions that can be generated according to some specific data, in any possible circumstance [11]. In the framework of statistical mechanics (SM), Jaynes pioneered the use of these IT ideas in order to both (i) reformulate and (ii) generalize the SM foundations [11]. In this paper we study Renyi properties in a MaxEnt environment.

It is well known that Renyi's entropic functional is not trace form. For all trace form functionals $F$, it has been shown in [12] that they, together with the MaxEnt structures, are able to reproduce the mathematical Legendre-invariant structures of thermodynamics. Thus, one may speak of an " $F$ thermodynamics." Of course, this is not guaranteed in the Renyi case, due to its lack of trace-class nature. In this paper we carefully investigate the issue further and conclude that there is no Renyi-associated thermodynamics. The main culprit of this Renyi failure is a hidden relation involving the Renyi's MaxEnt-Lagrange multipliers.

The paper is organized as follows: Section II deals with the conventional Renyi's MaxEnt treatment and compares it with that of Tsallis. Section III discusses some Renyi's MaxEnt thermodynamic troubles, while Sec. IV deals with the hidden constraint referred to above. Section V illustrates our ideas with reference to a simple, analytically tractable problem, while some conclusions are drawn in Sec. VI.

\section{CONVENTIONAL MaxEnt TREATMENTS}

\section{A. Renyi's MaxEnt}

Renyi's $S_{R}$ is defined as [9]

$$
S_{R}=\frac{1}{1-\alpha} \ln \left(\int_{M} P^{\alpha} d \mu\right),
$$

and the accompanying (canonical ensemble) MaxEnt probability distribution $P$ arises from the maximization of the functional $F_{S_{R}}(P)$ (where $U$ denotes the energy and $\langle U\rangle$ its mean value)

$$
\begin{aligned}
F_{S_{R}}(P)= & \frac{1}{1-\alpha} \ln \left(\int_{M} P^{\alpha} d \mu\right)+\lambda_{1}\left(\int_{M} P U d \mu-\langle U\rangle\right) \\
& +\lambda_{2}\left(\int_{M} P d \mu-1\right) .
\end{aligned}
$$

Following standard procedure we consider the functional- $h$ increment $[13,14]$

$$
\begin{aligned}
F_{S_{R}}(P+h)= & \frac{1}{1-\alpha} \ln \left[\int_{M}(P+h)^{\alpha} d \mu\right] \\
& +\lambda_{1}\left[\int_{M}(P+h) U d \mu-\langle U\rangle\right] \\
& +\lambda_{2}\left[\int_{M}(P+h) d \mu-1\right],
\end{aligned}
$$

so that

$$
\begin{aligned}
F_{S_{R}}(P+h)-F_{S_{R}}(P)= & \frac{1}{1-\alpha} \ln \left[\int_{M}(P+h)^{\alpha} d \mu\right] \\
& -\frac{1}{1-\alpha} \ln \left(\int_{M} P^{\alpha} d \mu\right) \\
& +\lambda_{1} \int_{M} h U d \mu+\lambda_{2} \int_{M} h d \mu .
\end{aligned}
$$


We now tackle $h^{2}$ contributions so as to assess second variations of $F_{S_{R}}[13,14]$ :

$$
\begin{aligned}
F_{S_{R}}(P+h)-F_{S_{R}}(P)= & \frac{1}{1-\alpha} \ln \left\{\int_{M}\left[P^{\alpha}+\alpha h P^{\alpha-1}+\frac{\alpha(\alpha-1)}{2} h^{2} P^{\alpha-2}\right] d \mu\right\} \\
& -\frac{1}{1-\alpha} \ln \left(\int_{M} P^{\alpha} d \mu\right)+\lambda_{1} \int_{M} h U d \mu+\lambda_{2} \int_{M} h d \mu,
\end{aligned}
$$

or, equivalently,

$$
F_{S_{R}}(P+h)-F_{S_{R}}(P)=\frac{1}{1-\alpha} \ln \left\{1+\frac{\int_{M}\left[\alpha h P^{\alpha-1}+\frac{\alpha(\alpha-1)}{2} h^{2} P^{\alpha-2}\right] d \mu}{\int_{M} P^{\alpha} d \mu}\right\}+\lambda_{1} \int_{M} h U d \mu+\lambda_{2} \int_{M} h d \mu,
$$

so that one finally arrives at

$$
\begin{aligned}
F_{S_{R}}(P+h)-F_{S_{R}}(P)= & \frac{1}{1-\alpha} \frac{\int_{M}\left[\alpha h P^{\alpha-1}+\frac{\alpha(\alpha-1)}{2} h^{2} P^{\alpha-2}\right] d \mu}{\int_{M} P^{\alpha} d \mu} \\
& -\frac{1}{2(1-\alpha)}\left(\frac{\int_{M} \alpha h P^{\alpha-1} d \mu}{\int_{M} P^{\alpha} d \mu}\right)^{2}+\lambda_{1} \int_{M} h U d \mu+\lambda_{2} \int_{M} h d \mu .
\end{aligned}
$$

Summing up, we have for the first variation

$$
\frac{\alpha}{1-\alpha} \frac{P^{\alpha-1}}{\int_{M} P^{\alpha} d \mu}+\lambda_{1} U+\lambda_{2}=0 .
$$

Functional calculus teaches that for the second variation one must demand [14]

$$
-\frac{1}{(1-\alpha)}\left(\frac{\int_{M} \alpha h P^{\alpha-1} d \mu}{\int_{M} P^{\alpha} d \mu}\right)^{2}-\alpha \frac{\int_{M} P^{\alpha-2} h^{2} d \mu}{\int_{M} P^{\alpha} d \mu} \leqslant C\|h\|^{2},
$$

with $C$ an arbitrary negative constant [14]. One must remember that functional calculus is not identical to ordinary calculus (involving ordinary functions), particularly when one is looking for extremes $[13,14]$.

The solution to $(2.8)$ is ( $Z$ below denotes Renyi's partition function and $\beta$ the inverse temperature $1 / T$ )

$$
\begin{gathered}
\lambda_{1}=\beta(\alpha-1) ; \quad \lambda_{2}=-1 ; \quad \alpha<1, \\
\lambda_{1}=\beta(1-\alpha) ; \quad \lambda_{2}=1 ; \quad \alpha>1, \\
Z=\int_{M}[1+(1-\alpha) \beta U]^{1 /(\alpha-1)} d \mu, \\
P=\frac{1}{Z}[1+(1-\alpha) \beta U]^{1 /(\alpha-1)} .
\end{gathered}
$$

A more detailed analysis of the relations of $\langle U\rangle$ with $\beta$ and $\alpha$ will be given in Sec. IV.

As for the second variation, we specialize things to positivedefinite Hamiltonians (quadratic, for instance) and restrict ourselves to scenarios with $\alpha<1$.

$$
\begin{aligned}
& -\frac{1}{(1-\alpha)}\left(\frac{\int_{M} \alpha h P^{\alpha-1} d \mu}{\int_{M} P^{\alpha} d \mu}\right)^{2}-\alpha \frac{\int_{M} P^{\alpha-2} h^{2} d \mu}{\int_{M} P^{\alpha} d \mu} \\
& \leqslant-\frac{\alpha}{\gamma} \int_{M} P^{\alpha-2} h^{2} d \mu \\
& \leqslant-\frac{\alpha}{\gamma} Z^{2-\alpha} \int_{M}[1+(1-\alpha) \beta U]^{(\alpha-2) /(\alpha-1)} h^{2} d \mu
\end{aligned}
$$

$$
\begin{aligned}
& \leqslant-\frac{\alpha}{\gamma} Z^{2-\alpha} \int_{M} h^{2} d \mu \\
& =-\frac{\alpha}{\gamma} Z^{2-\alpha}\|h\|^{2} \leqslant C\|h\|^{2},
\end{aligned}
$$

where $\gamma=\int_{M} P^{\alpha} d \mu$. It is clear that we can choose $C$ in the fashion

$$
-\frac{\alpha}{\gamma} Z^{2-\alpha}=C
$$

Some new results emerge already at this level. We see that restricting ourselves to (i) positive-definite Hamiltonians and (ii) $\alpha<1$, the MaxEnt functional $F_{S_{R}}$ attains always a maximum. The novelty here resides that in the cases of both (A) the above-mentioned restrictions (i) and (ii), and (B) arbitrary Hamiltonians and $\alpha$ 's, (2.9) must be investigated on a case-by-case basis. Nothing can be stated a priori regarding the existence, or not, of a MaxEnt maximum, contrary to popular belief.

\section{B. Comparison with well known results for Tsallis' MaxEnt}

During more than two decades, an important topic in statistical mechanics theory revolved around the notion of generalized $q$ statistics, pioneered by Tsallis [15]. It has been amply demonstrated that, in many circumstances, the Boltzmann-Gibbs-Shannon logarithmic entropy does not yield a correct description of the system under scrutiny [16]. Other entropic forms, called $q$ entropies, produce a much better performance [16]. One may cite a large number of such instances. For example, nonergodic systems exhibiting a complex dynamics [16]. The nonextensive statistical mechanics of Tsallis has been employed in many different areas of scientific endeavor [17].

The topic of Tsallis' variational problem has been discussed repeatedly in hundreds of papers (see, for instance, $[16,17]$ ). We discuss it below just for didactic purposes. The pertinent materials are, of course, not original. Tsallis's entropic functional is both trace form and a monotonous function of 
$S_{R}$. The associated MaxEnt functional reads [15]

$$
\begin{aligned}
F_{S_{T}}(P)= & \frac{1-\int_{M} P^{q} d \mu}{q-1}+\lambda_{1}\left(\int_{M} P U d \mu-\langle U\rangle\right) \\
& +\lambda_{2}\left(\int_{M} P d \mu-1\right),
\end{aligned}
$$

so that Tsallis' MaxEnt functional's first increment becomes

$$
\begin{aligned}
F_{S_{T}}(P+h)-F_{S_{T}}(P)= & \frac{1-\int_{M}(P+h)^{q} d \mu}{1-q} \\
& +\lambda_{1} \int_{M} h U d \mu \lambda_{2} \int_{M} h d \mu \\
& +\frac{1-\int_{M} P^{q} d \mu}{q-1} .
\end{aligned}
$$

The second order (in $h$ ) for this MaxEnt functional is

$$
\begin{aligned}
& F_{S_{T}}(P+h)-F_{S_{T}}(P) \\
& =\int_{M}\left[\left(\frac{q}{1-q}\right) P^{q-1}+\lambda_{1} U+\lambda_{2}\right] h d \mu \\
& \quad-\int_{M} q P^{q-2} \frac{h^{2}}{2} d \mu .
\end{aligned}
$$

From (2.17) we get

$$
\begin{gathered}
\left(\frac{q}{1-q}\right) P^{q-1}+\lambda_{1} U+\lambda_{2}=0 \\
-\int_{M} q P^{q-2} h^{2} d \mu \leqslant C\|h\|^{2} .
\end{gathered}
$$

The solution to (2.18) is

$$
\begin{gathered}
\lambda_{1}=-\beta q Z_{T}^{1-q}, \\
\lambda_{2}=\frac{q}{q-1} Z_{T}^{1-q}, \\
P=\frac{[1+\beta(1-q) U]^{1 /(q-1)}}{Z_{T}}, \\
Z_{T}=\int_{M}[1+\beta(1-q) U]^{1 /(q-1)} d \mu .
\end{gathered}
$$

Note that Eqs. (2.9) and (2.19) differ just in a constant. Consequently, Renyi's and Tsallis' maxima coincide. For a positive-definite Hamiltonian we have

$$
\begin{aligned}
& -\int_{M} q P^{q-2} h^{2} d \mu \\
& =-\int_{M} q Z_{T}^{2-q}[1+\beta(1-q) U]^{(q-2) /(q-1)} h^{2} d \mu \\
& \leqslant-q Z_{T}^{2-q}\left\|h^{2}\right\| \leqslant C\left\|h^{2}\right\|, \\
& -q Z_{T}^{2-q}=C,
\end{aligned}
$$

so that, for $q=\alpha$, the bound $C$ is the same in the two entropic instances.

\section{RENYI'S MaxEnt'S THERMODYNAMIC TROUBLES}

Let us express $S_{R}$ in terms of $Z$ and $\langle U\rangle$. To this end we replace in Eq. (2.8) for the first variation:

$$
\frac{\alpha}{1-\alpha} \frac{P^{\alpha-1}}{\int_{M} P^{\alpha} d \mu}+\lambda_{1} U+\lambda_{2}=0
$$

the values of $\lambda_{1}$ and $\lambda_{2}$ given by (2.10) and for the $P$ expression (2.12) (for $\alpha<1$ ).

$$
\begin{array}{r}
\frac{\alpha}{1-\alpha} \frac{1+\beta(1-\alpha) U}{Z^{\alpha-1} \int_{M} P^{\alpha} d \mu}+\lambda_{1} U+\lambda_{2}=0, \\
\frac{\alpha}{1-\alpha} \frac{1+\beta(1-\alpha) U}{Z^{\alpha-1} \int_{M} P^{\alpha} d \mu}-\beta(1-\alpha) U-1=0 .
\end{array}
$$

From the last relation one easily obtains

$$
\frac{\alpha Z^{1-\alpha}}{1-\alpha}=\int_{M} P^{\alpha} d \mu
$$

Thus we have for $S_{R}$,

$$
S_{R}=\ln Z+\frac{1}{1-\alpha} \ln \left(\frac{\alpha}{1-\alpha}\right) .
$$

Analogously, for $\alpha>1$ we find

$$
S_{R}=\ln Z+\frac{1}{1-\alpha} \ln \left(\frac{\alpha}{\alpha-1}\right) .
$$

We realize that in both instances (i) $S_{R}$ does not explicitly depend upon $\langle U\rangle$ and (ii) is not defined for $\alpha \rightarrow 1$, both troublesome results. In particular, as we shall see in great detail below, one expects the (canonical ensemble) entropy to be a sum of two terms. One of them contains the logarithm of the partition function. The other is $\beta\langle U\rangle$. This does not happen for $S_{R}$, according to Eq. (3.6).

Instead, for the Tsallis entropy we have from (2.18):

$$
\left(\frac{q}{1-q}\right) P^{q-1}-q \beta Z_{T}^{1-q} U+\frac{q}{q-1} Z_{T}^{1-q}=0,
$$

which, multiplied by $P$ yields

$$
\frac{P^{q}}{1-q}-\beta Z_{T}^{1-q} U P+\frac{P}{q-1} Z_{T}^{1-q}=0 .
$$

The last ratio can be recast as

$$
\frac{P^{q}-P}{1-q}-\beta Z_{T}^{1-q} U P+\frac{P}{q-1} Z_{T}^{1-q}+\frac{P}{1-q}=0,
$$

which can be integrated to yield

$$
S_{T}-\beta Z_{T}^{1-q}\langle U\rangle+\frac{1}{q-1} Z_{T}^{1-q}+\frac{1}{1-q}=0,
$$

or, equivalently,

$$
S_{T}-\beta Z_{T}^{1-q}\langle U\rangle-\frac{Z_{T}^{1-q}-1}{1-q}=0,
$$


so that $S_{T}$ becomes, invoking the so-called $q$ logarithm $\ln _{q}[16,18]$,

$$
S_{T}=\ln _{q} Z_{T}+\beta Z_{T}^{1-q}\langle U\rangle,
$$

which does exist in the limit $q \rightarrow 1$, where we encounter

$$
S=\ln Z_{\mathrm{BG}}+\beta\langle U\rangle,
$$

the usual thermodynamic Boltzmann-Gibbs relation. This crucial relationship that exists both in the BG and Tsallis cases cannot be reproduced à la Renyi, which constitutes a new result.

\section{THE HIDDEN RENYI-MaxEnt RELATION}

We have seen above that, in the MaxEnt framework, both Tsallis and Renyi functionals display the same extremes. This is due to the fact that Renyi's functional monotonously depends on Tsallis', as is well known [14]. However, these assertions lose some strength if one studies more closely Eq. (2.8), which we repeat below:

$$
\frac{\alpha}{1-\alpha} \frac{P^{\alpha-1}}{\int_{M} P^{\alpha} d \mu}+\lambda_{1} U+\lambda_{2}=0 .
$$

Indeed, multiplying it by $P$ we find

$$
\frac{\alpha}{1-\alpha} \frac{P^{\alpha}}{\int_{M} P^{\alpha} d \mu}+\lambda_{1} P U+\lambda_{2} P=0 .
$$

Integrating now we are led to

$$
\frac{\alpha}{1-\alpha}+\lambda_{1}\langle U\rangle+\lambda_{2}=0 \text {. }
$$

This is an important result, showing that $\lambda_{1}$ and $\lambda_{2}$ are not independent Lagrange multipliers, as MaxEnt assumes. We are authorized to write

$$
\lambda_{2}=\frac{\alpha}{\alpha-1}-\lambda_{1}\langle U\rangle,
$$

and replacing this value of $\lambda_{2}$ in (2.8) we get

$$
\frac{\alpha}{1-\alpha} \frac{P^{\alpha-1}}{\int_{M} P^{\alpha} d \mu}+\lambda_{1}(U-\langle U\rangle)+\frac{\alpha}{\alpha-1}=0,
$$

whose solution is given by

$$
\begin{gathered}
\lambda_{1}=-\beta \alpha, \\
P=\frac{[1+\beta(1-\alpha)(U-\langle U\rangle)]^{1 /(\alpha-1)}}{Z}, \\
Z=\int_{M}[1+\beta(1-\alpha)(U-\langle U\rangle)]^{1 /(\alpha-1)} d \mu .
\end{gathered}
$$

Using (4.7), the second variation equation (2.9) becomes

$$
\begin{aligned}
& -\frac{1}{(1-\alpha)}\left(\frac{\int_{M} \alpha h P^{\alpha-1} d \mu}{\int_{M} P^{\alpha} d \mu}\right)^{2}-\alpha \frac{\int_{M} P^{\alpha-2} h^{2} d \mu}{\int_{M} P^{\alpha} d \mu} \\
& \quad \leqslant-\frac{\alpha}{\gamma} \int_{M} P^{\alpha-2} h^{2} d \mu \\
& \quad \leqslant-\int_{M} \frac{\alpha}{\gamma} Z^{2-\alpha}[1+\beta(1-\alpha)(U-\langle U\rangle)]^{(\alpha-2) /(\alpha-1)} h^{2} d \mu \\
& \quad \leqslant C\left\|h^{2}\right\| .
\end{aligned}
$$

At this stage, two important new results ensue. Contrarily to what happened in Sec. II, we cannot assert now that, for a positive-definite Hamiltonian, the Renyi functional exhibits a MaxEnt maximum for $\alpha<1$.

Even worse, within the MaxEnt framework, including all its heavy armry, Renyi's expression is no longer a monotonous function of the Tsallis one, as it is outside the MaxEnt framework. This is so because within MaxEnt all entropic functionals become sums of products between Lagrange multipliers and so-called relevant observables. This sum is quite different in the Tsallis and the Renyi instances, as we have just seen.

Repeating now the steps of the preceding section so as to encounter a thermodynamic relation between $S_{R}, Z$, and $\langle U\rangle$ we find, starting with (4.1),

$$
\begin{gathered}
\frac{P^{\alpha-1}}{\int_{M} P^{\alpha} d \mu}+\frac{1-\alpha}{\alpha} \lambda_{1}(U-\langle U\rangle)-1=0, \\
\frac{P^{\alpha-1}}{\int_{M} P^{\alpha} d \mu}=1+\frac{\alpha-1}{\alpha} \lambda_{1}(U-\langle U\rangle) .
\end{gathered}
$$

Now we use (4.7) to arrive at

$$
\frac{1+\frac{\alpha-1}{\alpha} \lambda_{1}(U-\langle U\rangle)}{Z^{\alpha-1} \int_{M} P^{\alpha} d \mu}=1+\frac{\alpha-1}{\alpha} \lambda_{1}(U-\langle U\rangle) .
$$

From Eq. (4.12) we get

$$
\begin{gathered}
\int_{M} P^{\alpha} d \mu=Z^{1-\alpha}, \\
S_{R}=\frac{1}{1-\alpha} \ln \left(\int_{M} P^{\alpha} d \mu\right)=\frac{1}{1-\alpha} \ln \left[Z^{1-\alpha}\right],
\end{gathered}
$$

and, finally, the rather surprising relation

$$
S_{R}=\ln Z \text {, }
$$

an important new result. The essential link between statistical mechanics and thermodynamics is the relation between the entropy, $\beta\langle U\rangle$, and the logarithm of the partition function, the relation that defines Helmholtz' free energy. This is lost here, entailing that there is no Renyi thermodynamics.

Without the hidden constraint, the $S_{R}$-MaxEnt probabilities and partition function are given by, respectively, Eqs. (2.11) and (2.12), which are the equations employed in the literature. But the hidden constraint changes this situation to Eqs. (4.7) and (4.8), with devastating thermodynamic consequences.

Further, from (4.15) we realize that $S_{R}$ does not reduce to the Boltzmann-Gibbs entropy for $\alpha \rightarrow 1$.

\section{TWO-LEVEL MODEL FOR FIXED $\alpha=q=2$}

As an illustration we consider a two-level model with $U_{1}=$ $0, U_{2}=1$, and $\alpha=q=2$. From (2.11) and (2.12) we obtain

$$
Z=2-\beta ; \quad P_{1}=\frac{1}{2-\beta} ; \quad P_{2}=\frac{1-\beta}{2-\beta},
$$


and we get [see Eq. (2.11)]

$$
\begin{aligned}
& S_{R}=-\ln \left[\left(\frac{1}{2-\beta}\right)^{2}+\left(\frac{1-\beta}{2-\beta}\right)^{2}\right], \\
& S_{T}=1-\left[\left(\frac{1}{2-\beta}\right)^{2}+\left(\frac{1-\beta}{2-\beta}\right)^{2}\right] .
\end{aligned}
$$

From (5.2) and (5.3) we see that $S_{R}$ and $S_{T}$ display the same maxima.

Instead, if we consider Renyi's hidden relation one must use Eqs. (4.7) and (4.8) to deduce the expressions

$$
\begin{aligned}
Z & =2+2 \beta P_{2}-\beta, \\
P_{1} & =\frac{1+\beta P_{2}}{2+2 \beta P_{2}-\beta}, \\
P_{2} & =\frac{1+\beta P_{2}-\beta}{2+2 \beta P_{2}-\beta} .
\end{aligned}
$$

From (5.6) we obtain a quadratic equation for $P_{2}$, with two solutions, one of which leads to a negative $P_{2}$ and becomes inadmissible. Accordingly, for

$$
P_{2}^{2}+\frac{(1-\beta) P_{2}}{\beta}+\frac{\beta-1}{2 \beta}=0,
$$

we are left with the solution

$$
P_{2}=\frac{\sqrt{\beta^{2}-1}+\beta-1}{2 \beta},
$$

so that, after suitable replacement, we obtain

$$
P_{1}=\frac{\sqrt{\beta^{2}-1}+\beta+1}{2 \sqrt{1-\beta^{2}}+1} .
$$

Finally, the entropy becomes

$S_{R}=-\ln \left[\left(\frac{\sqrt{\beta^{2}-1}+\beta+1}{2 \sqrt{1-\beta^{2}}+1}\right)^{2}+\left(\frac{\sqrt{\beta^{2}-1}+\beta-1}{2 \beta}\right)^{2}\right]$

which (i) is not a monotone function of Tsallis' entropy, and (ii) does not display the Tsallis maxima.

\section{CONCLUSIONS}

We studied in great detail the possible existence of a Renyi's thermodynamics, with negative results. Summing up:

(a) As a first result we saw that for (i) positive definite Hamiltonians and (ii) $\alpha<1$, the MaxEnt functional $F_{S_{R}}$ always attains a maximum. The novelty here resides in

(1) point (i) above and the $\alpha$ restriction (ii);

(2) for arbitrary Hamiltonians and $\alpha$ 's, (2.9) must be investigated on a case-by-case basis. Nothing can be stated $a$ priori regarding the existence, or not, of a MaxEnt maximum, contrary to popular belief.

(b) $S_{R}$ does not explicitly depend upon $\langle U\rangle$ and is not defined for $\alpha \rightarrow 1$, both troublesome results.

(c) The relation

$$
S=\ln Z_{\mathrm{BG}}+\beta\langle U\rangle
$$

is a crucial thermodynamic Boltzmann-Gibbs relation. This critical relationship that exists both in the BG and Tsallis cases cannot be reproduced à la Renyi (because $S_{R}=\ln Z$ ), which constitutes a new result.

(d) The hidden $S_{R}$-MaxEnt relation

$$
\lambda_{2}=\frac{\alpha}{\alpha-1}-\lambda_{1}\langle U\rangle,
$$

linking $\alpha,\langle U\rangle$, and the two Lagrange multipliers, is a crucial new result.

(e) As a consequence, contrarily to what happened in Sec. II, we cannot assert that, for a positive-definite Hamiltonian, the Renyi functional exhibits a MaxEnt maximum for $\alpha<1$. Even worse, within the MaxEnt framework Renyi's expression is no longer a monotonous function of the Tsallis one. Without the hidden constraint, the $S_{R}$ 's MaxEnt probabilities and partition function are given by, respectively, Eqs. (2.11) and (2.12), which are the equations employed in the literature. But the hidden constraint changes this situation to Eqs. (4.7) and (4.8), with devastating thermodynamic consequences.

Finally, let us insist: the essential link between statistical mechanics and thermodynamics is the relation between the entropy, $\beta\langle U\rangle$, and the logarithm of the partition function, the relation that defines Helmholtz' free energy. This is lost here, entailing that there is no Renyi thermodynamics.

However, it must be stated that our discovery does not invalidate the use of Renyi concepts to study quantum entanglement or as a tool to guide quantum Monte Carlo calculations.
[1] C. M. Herdman, S. Inglis, P.-N. Roy, R. G. Melko, and A. Del Maestro, Phys. Rev. E 90, 013308 (2014).

[2] M. H. Ansari and Yuli V. Nazarov, Phys. Rev. B 91, 174307 (2015).

[3] L. Wang and M. Troyer, Phys. Rev. Lett. 113, 110401 (2014).

[4] M. B. Hastings, I. González, A. B. Kallin, and R. G. Melko, Phys. Rev. Lett. 104, 157201 (2010).

[5] R. Berkovits, Phys. Rev. Lett. 115, 206401 (2015).

[6] N. Lashkari, Phys. Rev. Lett. 113, 051602 (2014).

[7] G. B. Halasz and A. Hamma, Phys. Rev. Lett. 110, 170605 (2013).
[8] A. De Gregorio and S. M. lacus, Inf. Sci. 179, 279 (2009).

[9] L. Golshani, E. Pasha, and G. Yari, Inf. Sci. 179, 2426 (2009); J. F. Bercher, ibid. 178, 2489 (2008).

[10] E. K. Lenzi, R. S. Mendes, and L. R. da Silva, Physica A 280, 337 (2000).

[11] E. T. Jaynes, Phys. Rev. 106, 620 (1957); 118, 171 (1961); Papers on Probability, Statistics and Statistical Physics, edited by R. D. Rosenkrantz (Reidel, Dordrecht, Holland, 1983); L. Brillouin, Science and Information Theory (Academic, New York, 1956); W. T. Grandy, Jr. and P. W. Milonni, Physics 
and Probability: Essays in Honor of E. T. Jaynes (Cambridge University Press, Cambridge, England, 1993).

[12] A. Plastino and A. R. Plastino, Phys. Lett. A 226, 257 (1997).

[13] A. Plastino and M. C. Rocca, Physica A 436, 572 (2015).

[14] G. Y. Shilov, Mathematical Analysis (Pergamon, New York, 1965).

[15] C. Tsallis, J. Stat. Phys. 52, 479 (1988).
[16] C. Tsallis, Introduction to Nonextensive Statistical Mechanics: Approaching a Complex World (Springer, New York, 2009).

[17] C. M. Gell-Mann and C. Tsallis, Nonextensive Entropy: Interdisciplinary Applications (Oxford University Press, New York, 2004).

[18] The $q$ logarithm is defined in the fashion [16] $\ln _{q} Z=\frac{Z^{1-q}-1}{1-q}$. 